\title{
Synchronization in a population of globally coupled chaotic oscillators
}

\author{
A. S. Pikovsky, M. G. Rosenblum $(*)$ and J. Kurths \\ Max-Planck-Arbeitsgruppe "Nichtlineare Dynamik" an der Universität Potsdam \\ Am Neuen Palais 19, PF 601553, D-14415 Potsdam, Germany (**)
}

(received 30 November 1995; accepted in final form 9 March 1996)

PACS. $05.45+\mathrm{b}-$ Theory and models of chaotic systems.

PACS. 64.60Cn - Order-disorder and statistical mechanics of model systems.

\begin{abstract}
We demonstrate synchronization transition in a large ensemble of non-identical chaotic oscillators, globally coupled via the mean field. We show that this coherent behaviour is due to synchronization of phases of these oscillators, while their amplitudes remain chaotic. Two types of transition, depending on the phase coherence properties of the individual systems, are described.
\end{abstract}

A number of physical, chemical and biological systems can be viewed as large ensembles of weakly interacting non-identical oscillators [1]. One of the most popular models here is an ensemble of globally coupled non-linear oscillators. Such systems appear in the studies of Josephson junction arrays [2], oscillatory neuronal systems [3], multimode lasers [4], chargedensity waves [5], etc. Investigations of ensembles of non-linear continuous-time oscillators have revealed such interesting phenomena as clustering [6], existence of splay states [7], finite-sizeinduced transition [8], dephasing and bursting [9] and collective chaotic behaviour [6], [10]. A non-trivial transition to self-synchronization in a population of periodic oscillators with different natural frequencies coupled through a mean field has been described by Kuramoto [11]. In this system, as the coupling parameter increases, a sharp transition is observed for which the mean-field intensity serves as an order parameter. This transition owes to a mutual synchronization of the oscillators, so that their fields become coherent (i.e. their phases are locked), thus producing a macroscopic mean field. In its turn, this field acts on the individual oscillators, locking their phases, so that the synchronous state is self-sustained. Different aspects of this transition have been studied in [12], where also an analogy with a second-order phase transition has been exploited.

In this letter we describe self-synchronization transitions in a population of chaotic systems. We explain this by the recently found phenomenon of phase synchronization of chaotic oscillators [13].

As a basic model we consider a population of non-identical Rössler oscillators

$$
\left\{\begin{array}{l}
\dot{x}_{i}=-\omega_{i} y_{i}-z_{i}+\varepsilon X, \\
\dot{y}_{i}=\omega_{i} x_{i}+a y_{i}, \\
\dot{z}_{i}=0.4+z_{i}\left(x_{i}-8.5\right),
\end{array}\right.
$$

$\left({ }^{*}\right)$ A. von Humboldt Fellow. Permanent address: Mech. Eng. Res. Institute, Russian Academy of Sciences, Moscow, Russia.

$\left({ }^{* *}\right)$ Homepage: www.agnld.uni-potsdam.de.

(C) Les Editions de Physique 


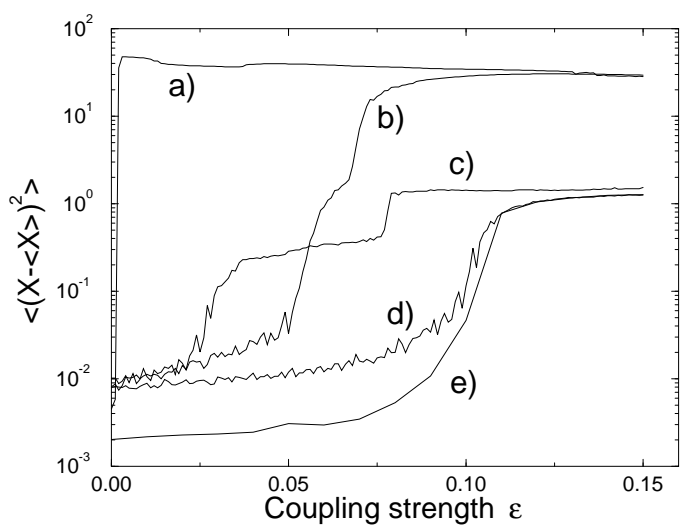

Fig. 1. - Variance of the mean field $X$ vs. coupling parameter $\varepsilon$ for different topologies of the Rössler system $\left(a=0.15, \omega_{0}=1\right.$ for curves $\left.a\right)$ and $\left.b\right) ; a=0.25, \omega_{0}=0.97$ for curves $c$ ) and $\left.d\right)$ ) and different distributions of natural frequencies $(\Delta \omega=0$ for curves $a$ ) and $c$ ); $\Delta \omega=0.02$ for curves $b$ ) and $d)$ ). The number of oscillators is $N=5000$ for curves $a$ )- $d$ ). Curve $e$ ) differs from curve $d$ ) only in the size of the ensemble $(N=20000)$; it demonstrates the finite-size effect on the order parameter.

coupled via the mean field $X=N^{-1} \sum_{1}^{N} x_{i}$. Here $N$ is the number of elements in the ensemble, $\varepsilon$ is the coupling constant, $a$ and $\omega_{i}$ are parameters of the Rössler oscillators [14]. The parameter $\omega_{i}$ governs the natural frequency of an individual system [13]. We take a set of frequencies $\omega_{i}$ Gaussian distributed around the mean value $\omega_{0}$ with variance $(\Delta \omega)^{2}$. Because the Rössler system typically shows windows of periodic behaviour as the parameter $\omega$ is changed, we usually choose such a mean frequency $\omega_{0}$ that avoids large periodic windows. The parameter $a$ governs the topological type of the Rössler attractor; its significance is discussed below.

In our computer simulations we solve eqs. (1) numerically for rather large ensembles $N=$ 3000-5000. From our numerical calculations we have good indications that these ensembles are large enough to describe the dynamics correctly in the thermodynamic limit $N \rightarrow \infty$.

With an increase of the coupling strength $\varepsilon$, the appearance of a non-zero macroscopic mean field $X$ is observed, as is shown in fig. 1. There the order parameter (the variance of the mean field) is depicted vs. the coupling $\varepsilon$ for two values of the parameter $a$ of the Rössler system

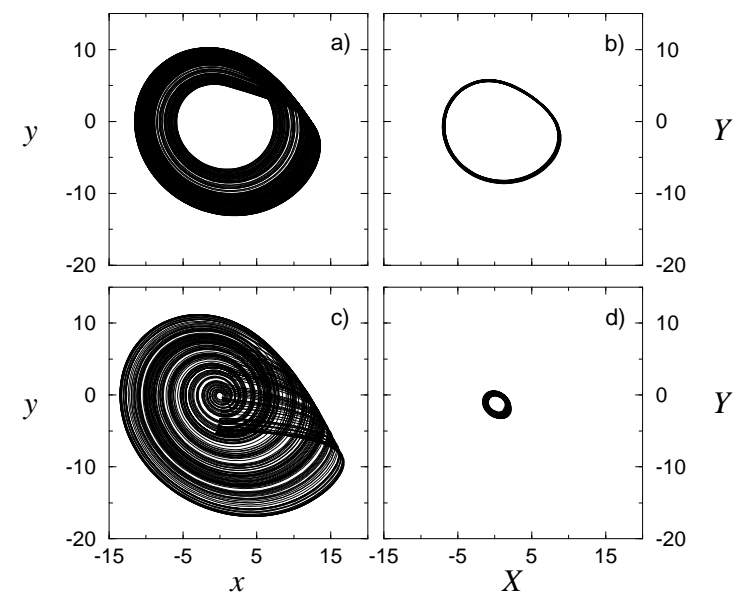

Fig. 2. - Projections of the phase portraits of the Rössler oscillators (left column) and of the mean fields $X=\left\langle x_{i}\right\rangle, \quad Y=\left\langle y_{i}\right\rangle$ in an ensemble of $N=5000$ oscillators. a) Phase-coherent Rössler attractor, $\omega_{0}=1, a=0.15$. b) Mean field in the ensemble of oscillators $a$ ) with Gaussian distribution of frequencies $\Delta \omega=0.02$ and coupling $\varepsilon=0.1$. c) Funnel attractor $\omega_{0}=0.97, a=0.25$. d) Mean field in the ensemble of oscillators $c$ ) with Gaussian distribution of frequencies $\Delta \omega=0.02$ and coupling $\varepsilon=0.15$. 

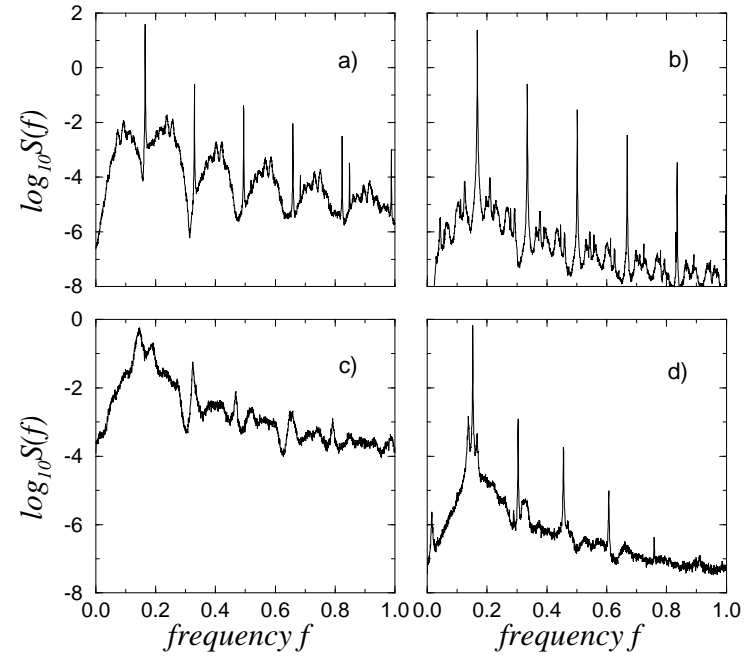

Fig. 3. - Power spectra of the processes $x(t)$ and $X(t)$ from fig. 2 .

and for ensembles of identical $(\Delta \omega=0)$ and non-identical $(\Delta \omega>0)$ oscillators. The non-zero value of the order parameter $(\approx 0.01)$ for small couplings is due to finite-size effects (finiteness of $N$, compare curves $d$ ) and $e$ )).

We first focus on the non-identical case (curves $b$ ) and $d)$ ). One can see that although qualitatively a macroscopic mean field appears for both values of the parameter $a$, for $a=0.15$ the field is much stronger than for $a=0.25$. Also the threshold $\varepsilon_{\mathrm{c}} \approx 0.05$ for $a=0.15$ is significantly smaller than $\varepsilon_{\mathrm{c}} \approx 0.1$ for $a=0.25$. We attribute this to the quite different topologies of the corresponding strange attractors and hence the corresponding phase coherence properties to be described below.

The phase portrait of the Rössler attractor for $a=0.15$ is shown in fig. $2 a$ ). Here, the motion can be well represented as oscillations with a chaotic amplitude modulation, while the dynamics of the phase is relatively regular. In the power spectrum of the variable $x(t)$, this manifests itself as a sharp peak above a broad-band component (fig. 3a)) [15], [16]. This type of attractor is called phase-coherent. In fact, we can introduce the phase of the Rössler attractor by making a projection of $(1)$ on the plane $(x, y)$ and taking the value of

$$
\phi_{i}(t)=\arctan \left(x_{i}(t) / y_{i}(t)\right)
$$

for the instantaneous phase. As has been argued in [17], [13], the dynamics of the phase is similar to that of a periodic oscillator and can be described by the following model equation:

$$
\dot{\phi}_{i}=\omega_{i}+F\left(A_{i}\right) .
$$

Here $\omega_{i}$ is the mean frequency of oscillations and $F(A)$ is the amplitude-dependent non-linear term responsible for the non-uniform phase motion. Due to the chaotic nature of the attractor, $F(A)$ can be considered as a noisy term responsible for the phase diffusion.

Regarding the mean field $X(t)$ in eq. (1) as an external force having nearly constant amplitude (which is confirmed by numerics, see fig. $2 b$ )) and the phase $\psi$, we can write the dynamics of the phase $\phi_{i}$ under this force as

$$
\dot{\phi}_{i}=\omega_{i}+F\left(A_{i}\right)+\varepsilon G\left(\phi_{i}, \psi\right),
$$

where $G$ is a $2 \pi$-periodic in each argument function describing phase entrainment by the external force; in the first approximation one can take $G(\phi, \psi) \sim \sin (\phi-\psi)$. Comparing with the model of globally coupled periodic noise-driven oscillators having distribution of natural 

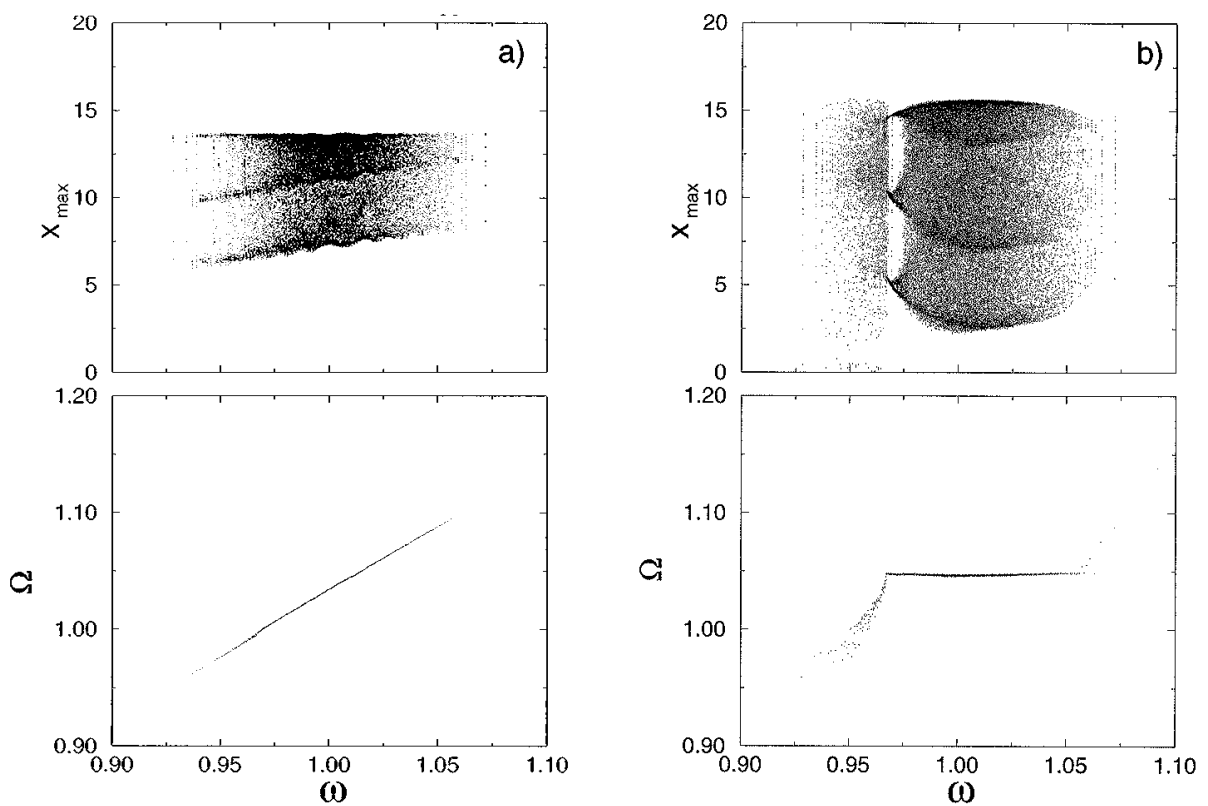

Fig. 4. - Successive maxima (upper panel) and observed frequencies, eq. (5) (bottom panel) vs. natural frequencies in the ensemble of coupled phase-coherent Rössler systems of fig. $2 a$ ). a) The coupling $\varepsilon=0.05$ is slightly below the transition threshold, the observed frequencies $\Omega$ are proportional to the natural frequencies $\omega . b)$ Above threshold $(\varepsilon=0.1)$ most of the oscillators form a coherent cluster (plateau in the bottom panel), while the amplitudes remain chaotic (with the exception of the period-3 window for $\omega \approx 0.97)$.

frequencies [18], we can see that the difference is in the term $F(A)$, which, instead of being Gaussian white noise, describes rather specific properties of phase dynamics in a particular chaotic system. Nevertheless, one can expect that qualitatively this term acts as an effective noise, thus allowing to consider the transition in the ensemble of chaotic autonomous oscillators as a phenomenon analogous to the synchronization transition in a network of coupled noisy limit-cycle oscillators.

In fact, for the Rössler attractor (fig. $2 a$ )) this term is rather small, so the phase can be easily locked by an external periodic force [17], [16] or due to the interaction of different oscillators [13]. Thus, the self-induced synchronization in the population of the Rössler systems can be explained as a Kuramoto-type transition in a network of oscillators without noise: the phases of some part of the ensemble become locked and the coherent summation of corresponding contributions leads to a non-zero mean field, while the amplitudes remain chaotic and uncorrelated. (A similar synchronization has been reported in [19] for a lattice of locally coupled identical Rössler systems.)

This is illustrated in fig. 4 where we plot the observed frequency $\Omega_{i}$ of the $i$-th oscillator, defined as the average derivative of the phase (2):

$$
\Omega_{i}=\left\langle\dot{\phi}_{i}\right\rangle=\left\langle\left(x_{i} \dot{y}_{i}-\dot{x}_{i} y_{i}\right)\left(x_{i}^{2}+y_{i}^{2}\right)^{-1}\right\rangle,
$$

$v s$. the natural frequency $\omega_{i}$.

In the absence of coupling $(\varepsilon=0)$ the observed frequency $\Omega_{i}$ is, as one could expect, proportional to $\omega_{i}$. With the increase of coupling we observe the appearance of a plateau in the plot, i.e. these averaged frequencies of a large number of oscillators become equal. Respectively, these systems oscillate in-phase, and their contributions to the mean field produce a non-zero component. In the upper panel of fig. 4, we plot the values of the maxima 


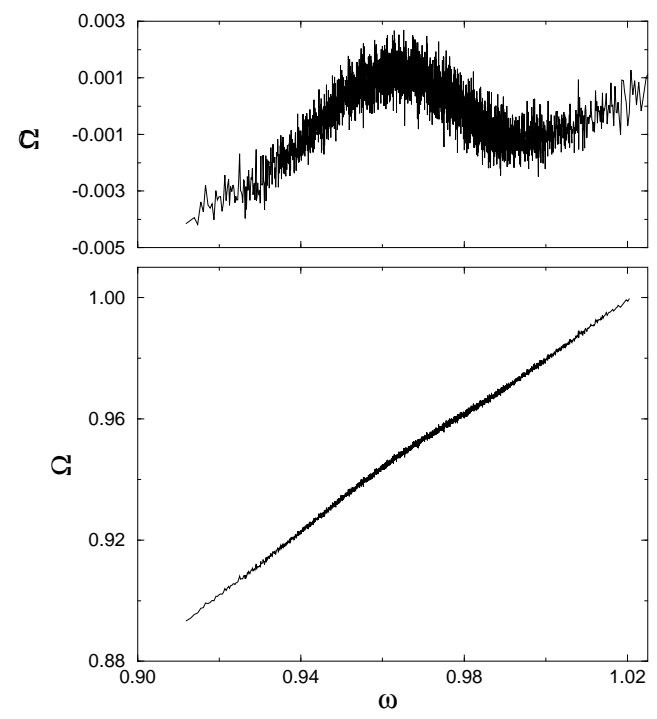

Fig. 5. - The observed frequency $\Omega$ in the ensemble of coupled funnel attractors with parameters of fig. $2 d$ ). The upper panel shows the deviation $\tilde{\Omega}$ from the linear fit: the tendency to synchronization is clearly seen in this panel, although it is rather small.

of the field $x_{i}$ for each oscillator. The distribution of these maxima gives an impression about the chaoticity of the amplitudes; we see that even in the case when almost all systems are synchronized, the amplitudes remain chaotic (with the exception of a small number of systems with periodic behaviour; it is worth noting that both chaotic and periodic systems are nevertheless synchronized). This agrees with recent findings [13], where the synchronization of two Rössler attractors has been considered and the chaoticity of the amplitudes has been verified by calculations of the Lyapunov exponents. Because the phases of different oscillators are locked, the mean field is fairly periodic, as demonstrated in a phase portrait (fig. $2 b)$ ), where $Y=N^{-1} \sum_{1}^{N} y_{i}$ is plotted $v s . X$ and in the power spectrum of the variable $X$ (fig. $3 b$ )). Some modulation of the mean field visible there seems to be a finite-size effect.

We now discuss the situation when the Rössler oscillator has a rather weak phase coherence, e.g. for $a=0.25$. The corresponding so-called funnel attractor [15], [16] is presented in fig. $2 c$ ) and the power spectrum of $x(t)$ in fig. $3 c$ ). The spectrum has no sharp peak because sometimes a trajectory makes a roundtrip around the origin in the $(x, y)$-plane, and sometimes it makes only a half of this roundtrip (fig. $2 c$ )). These irregular phase shifts can be interpreted as a large effective noise term $F(A)$ in eqs. (3), (4) which breaks the phase coherence. Nevertheless, for sufficiently large couplings a macroscopic highly coherent mean field appears (fig. 1, curve $d$ ); fig. $3 d)$ ), although this field is much smaller than in the case of the phase-coherent attractor (fig. $2 a)$ ). We interpret this transition as a synchronization transition, described for noisy coupled phase oscillators in [1], [18]. There it has been demonstrated that in an ensemble of globally coupled noisy phase oscillators a transition to a non-uniform distribution of the phases and to a macroscopic mean field occurs at a critical value of coupling. Similar transitions in coupled two-well noisy oscillators have been described in [20].

A qualitative difference between these two types of synchronization becomes clear if we consider the dependence of the observed frequency on the natural one. In the case of the phase-coherent attractor, the phases of the entrained oscillators are completely locked, and their observed frequencies coincide almost perfectly (fig. 4 b)). For funnel attractors there is no plateau in the distributions of frequencies; only a small "attraction" to the frequency of the mean field is seen (fig. 5). Exactly such an attraction occurs at the synchronization of 
a noisy oscillator by a periodic force [21]. Nevertheless, this attraction produces the visible macroscopic effect.

Another difference is that the synchronization of the phase-coherent oscillators occurs already for very small couplings, if the distribution of natural frequencies is narrow; for noisy oscillators even for identical natural frequencies a finite threshold of synchronization exists, depending on the noise strength. This is illustrated in fig. 1, where the mean field is shown for the ensemble of identical systems $(\Delta \omega=0)$ with phase-coherent (curve $a)$ ) and funnel (curve $c$ )) attractors.

In conclusion, we have demonstrated that in a population of globally coupled chaotic oscillators a transition to phase synchronization can be observed. The order parameter for this transition is the intensity of the mean field. The features of the transition depend crucially on the phase coherence properties of the individual systems. If the chaotic oscillators are phase-coherent, i.e. have a sharp peak in the spectrum, the dynamics of the phase is very similar to that in the population of periodic oscillators, the amplitudes of the oscillators remain, however, chaotic. Such a transition is also observed for the systems with coupled non-phase-coherent funnel attractors. In the latter case it is similar to the transition in a population of noisy phase oscillators. Statistical properties of the mean field and the finite-size effects remain a problem for future studies.

We thank M. ZAKs for useful discussions. MGR acknowledges support from Alexander von Humboldt Foundation.

\section{REFERENCES}

[1] Kuramoto V., Chemical Oscillations, Waves and Turbulence (Springer, Berlin) 1984.

[2] Hadley P., Beasley M. R. and Wiesenfeld K., Phys. Rev. B, 38 (1988) 8712.

[3] Sompolinsky H., Golomb D. and Kleinfeld D., Phys. Rev. A, 43 (1991) 6990.

[4] Wiesenfeld K., Bracikowski C., James G. and Roy R., Phys. Rev. Lett., 65 (1990) 1749.

[5] Strogatz S. H., Marcus C. M., Westervelt R. M. and Mirollo R. E., Physica D, 36 (1989) 23.

[6] Golomb D., Hansel D., Shraiman B. and Sompolinsky H., Phys. Rev. A, 45 (1992) 3516.

[7] Nichols S. and Wiesenfeld K., Phys. Rev. A, 45 (1992) 8430; Strogatz S. H. and Mirollo R. E., Phys. Rev. E, 47 (1993) 220.

[8] Pikovsky A. S., Rateitschak K. and Kurths J., Z. Phys. B, 95 (1994) 541.

[9] Han S. K., Kurrer C. and Kuramoto Y., Phys. Rev. Lett., 75 (1995) 3190.

[10] Wiesenfeld K. and Hadley P., Phys. Rev. Lett., 62 (1989) 1335; Hakim V. and Rappel W.-J., Phys. Rev. A, 46 (1992) R7347; Nakagawa N. and Kuramoto Y., Prog. Theor. Phys., 89 (1993) 313.

[11] Kuramoto Y., Prog. Theor. Phys. Suppl., 79 (1974) 223; in International Symposium on Mathematical Problems in Theoretical Physics, edited by H. ARAKI (Springer, New York, N.Y.) 1975.

[12] Sakaguchi H., Shinomoto S. and Kuramoto Y., Prog. Theor. Phys., 77 (1987) 1005; DAido H., Prog. Theor. Phys., 75 (1986) 1460; DAido H., J. Stat. Phys., 60 (1990) 753.

[13] Rosenblum M., Pikovsky A. and Kurths J., Phys. Rev. Lett., 76 (1996) 1804.

[14] Rössler O. E., Phys. Lett. A, 57 (1976) 397.

[15] Crutchfield J. et al., Phys. Lett. A, 76 (1980) 1.

[16] Stone E. F., Phys. Lett. A, 163 (1992) 367.

[17] Pikovsky A. S., Sov. J. Commun. Technol. Electron., 30 (1985) 1970.

[18] Sakaguchi H., Prog. Theor. Phys., 79 (1988) 39; Bonilla L. L., Neu J. C. and Spigler R., J. Stat. Phys., 67 (1992) 313.

[19] Brunnet L., Chaté H. and Manneville P., Physica D, 78 (1994) 141.

[20] Desai R. C. and Zwanzig R., J. Stat. Phys., 19 (1978) 1.

[21] Stratonovich R. L., Topics in the Theory of Random Noise (Gordon and Breach, New York, N.Y.) 1963. 